\title{
Synthesis Load Forecasting Method Based on Artificial Immune System for Power System
}

\author{
Dong Nan ${ }^{1}, Z h u$ Haojun $^{1}, X i$ Yunhua $^{1}$ \\ ${ }^{1}$ China Southern Power Grid Energy Development Research Institute Co., Ltd., 510000 Guangzhou, China
}

\begin{abstract}
Electric power load forecasting is not only the sticking point of the safely, operation of whole system, but also the key part of the economical and healthy development of electric power system. The intrinsic single models have shortage, so the synthesis forecasting model making better use of all information will be pursued. It combines those single models property to take full advantage of their information to improve the precision. The most important part of the combination forecasting model is how to confirm the weight. In AIS, antigen and antibody are the parallelism of aim function and doable result. The appetency between antigen and antibody is regarded as the matching degree between feasible result and the objective function. Because of its good property on global searching, it can find the optimal solutions, some synthetic forecasting models based on AIS are set up in this paper, which combine AIS and load forecasting. The attempter average synthetic model and power geometry average synthetical model proposed in this paper, has been applied to a certain area mid-long term load forecasting. It is showed that the synthetic forecasting model based on AIS could provide high forecasting precision.
\end{abstract}

\section{Introduction}

In today's unprecedented development of China's electric power industry, power management is moving towards the market, and power load forecasting has become an important and arduous task we are facing ${ }^{[1]}$.

Although the existing traditional forecasting methods are relatively mature, different forecasting methods are put forward under certain assumptions. If we simply abandon some methods with large forecasting errors, some useful information will be lost. Prediction accuracy is affected, therefore, in the past decade, there has been a rise in the combination of different prediction methods, making full use of the useful information contained in each single prediction model, to improve the prediction accuracy of the comprehensive prediction research ${ }^{[2]}$.

The emphasis of comprehensive prediction is to determine the weight of each single prediction model. This paper will use artificial immune algorithm to study.

Artificial immune algorithm is a random search method based on the working mechanism of simulated biological immune system. It combines the two characteristics of prior knowledge and adaptive ability of biological immune system, so it has strong and robust information processing ability, and is similar to other intelligent algorithms. In the process of solving the problem, artificial immune algorithm combines the advantages of prior knowledge and adaptive ability of biological immune system. The objective function is not required to have derivable and other high additional information, so it is more efficient in the search process. As an important research branch of artificial immune system, the theory and application of immune algorithm has attracted close attention of computational intelligence experts and scholars at home and abroad.

In this paper, we hope that the artificial immune algorithm can be applied to the comprehensive forecasting of power load in order to establish a highprecision forecasting model and make a useful attempt to the application of artificial immune algorithm.

\section{Artificial Immune Algorithms}

\subsection{Overview of Artificial Immune Algorithms ${ }^{[3,4]}$}

Genetic algorithm is a heuristic algorithm based on multi-point random search, which has many advantages compared with traditional algorithm. However, in the application of genetic algorithm, there are often conflicting problems. For example, in the late iteration period, the number of individuals with large fitness in the population is absolutely dominant and lost. Individual diversity sometimes leads to prematurity and falls into local optimum. Although the premature population maturity can be avoided by increasing the crossover rate and mutation rate, too large crossover rate and mutation rate will reduce the search efficiency, thus reducing the convergence rate.

Considering these problems of genetic algorithm and the characteristics of immune system, a new algorithm has been discovered: artificial immune algorithm. Artificial immune algorithm research began in the late 1980 s, and its research is still in its infancy. Its idea

\footnotetext{
* Corresponding author: 1942223602@qq.com
} 
comes from the biological immune system, which simulates the learning and memory functions of the immune system to carry out pattern recognition and optimization search. The diversity regulation mechanism of biological immune system is applied to the algorithm design, so that the algorithm can avoid falling into local optimum in the search process.

\subsection{Basic principles of Artificial Immune Algorithms}

As an intelligent optimization algorithm, the basic process of AIA is very similar to that of genetic algorithm, i.e. through random initialization, chromosome evaluation and genetic operation, it can obtain better individuals (i.e. solution of the scheme).

Compared with other intelligent optimization algorithms, immune algorithm has the following advantages:

(1) Diversity is preserved: Because immune algorithm has two main characteristics, namely diversity and self-regulation, many optimal solutions of optimization problems can be obtained by using this method.

(2) Memory training: Using immune algorithm, the optimal solution can be obtained quickly through repeated optimization process. Because for the antigens that have appeared before, the immune algorithm produces the corresponding antibodies faster than before.

Artificial immune algorithm corresponds antigen and antibody to the objective function and feasible solution of optimization problem respectively. The affinity between antibody and antigen is regarded as the matching degree between feasible solution and objective function; the affinity between antibodies ensures the diversity of feasible solution, promotes the inheritance and variation of better antibody by calculating the expected survivability of antibody, and inhibits the continuation of similar feasible solution by preserving the preferred feasible solution in memory cell unit. At the same time, when the similar problem appears again, it can produce the optimal solution or even the optimal solution which adapts to the problem quickly. The theory proves that the artificial immune algorithm converges globally according to probability 1 .

\subsubsection{Antibody and antigen}

In biological immunology, the substance that induces immune system to produce immune response is called antibody, and the external harmful pathogen is called antigen. In AIS system theory, antibody and antiprinciple represent the feasible solution and objective function of the problem to be optimized respectively. Similar to genetic algorithm, AIS is used to model and calculate. First, the expression of the solution must be found. The method of antibody description is exactly similar to chromosome in genetic algorithm. It can be coded in binary, decimal or floating-point forms as needed.

\subsubsection{Affinity}

The affinity between antibody and antibody reflects the similarity of antibody. When the affinity is high, the more similar the antibody is. On the contrary, the more similar the antibody is. The affinity of antibody to antibody was calculated by the following formula:

$$
q_{u v}=\frac{1}{1+h_{u v}}
$$

The value of $q_{u v}$ is between 0 and 1, the smaller $q_{u v}$ is, the smaller the similarity between antibody and antibody. It represents the information entropy between two antibodies and can be expressed by the distance between the antibodies, indicating the length of the antibody gene.

The fitness value between antigen and antibody reflects the closeness of feasible solution to objective function, which is obtained by objective function transformation of optimization problem. The fitness values of antibodies and antigens are as follows:

$$
Q_{u}=\text { fittness }(u)
$$

There, $u$ is an antibody, fitness $(u)$ is the fitness function (objective function), the larger the value, the closer the antigen and antibody are, and the better the solution of the antibody.

\subsubsection{Concentration}

The formula for calculating antibody concentration is as follows:

$$
L(u)=\frac{1}{N} \sum_{j=1}^{N} c_{u j}
$$

Here :

$$
c_{u j}=\left\{\begin{array}{l}
1, q_{u j} \geq h_{2} \\
0, q_{u j}<h_{2}
\end{array} \quad j=1,2, \cdots N\right.
$$

Among them, $\mathrm{N}$ is the number of antibodies, is the set threshold of antibody affinity. The formula regards the antibodies satisfying a certain approximation as the same antibody. Antibody concentration is used to promote or inhibit the production of similar solutions, thereby regulating the maintenance of antibody diversity.

\subsubsection{Expected survival rate}

Antibody selection can be achieved by rotating the wheel according to the expected survival rate. The higher the expected survival rate, the more likely the antibody will be selected. On the contrary, the less likely it will be. Expected survival rate of Antibody $u$ is:

$$
P_{u}=\frac{Q u\left(1-r_{u}\right)}{\operatorname{Lu} \sum_{i=1}^{N} Q_{i}}
$$

Where:

$$
r_{u}=\left\{\begin{array}{l}
L_{u}, L_{u} \geq h_{2} \\
0, L_{u}<h_{2}
\end{array}\right.
$$

Among them, $h_{2}$ is the threshold of antibody concentration. When the fitness of antibody is high and 
the concentration is low, the probability of antibody selection is high, which fully reflects the better protection of antibody, at the same time, it promotes the antibody with lower concentration and ensures the understanding of the diversity of antibody in evolution.

\section{Integrated load forecasting of power system using AIS}

\subsection{Basic ideas ${ }^{[2,5-7]}$}

The essence of power load comprehensive forecasting is to deal with the weights of the forecasting results of multiple models. In view of the strong search ability of AIS, this paper tries to use artificial immune algorithm to optimize and determine the weights of the comprehensive forecasting model, and constructs a harmonic average comprehensive forecasting model.

The basic idea of the model is that the optimal weights of each single power load forecasting model in the comprehensive model of power load forecasting are used as antigens, the solution of weights is used as antibodies, and the working principle of biological immune system is simulated to search for the optimal weights, i.e. through the initialization of antibodies, the calculation of objective functions, the immune genetic evaluation and the immune genetic evaluation. The process of selection, crossover and mutation of immune genetic operation is used to search for the global optimal solution.

\subsection{Harmonic average comprehensive prediction model based on AIS}

Harmonic average comprehensive forecasting is a kind of non-linear comprehensive forecasting method. It can be described as follows:

$$
\hat{y} t=\frac{\sum_{j=1}^{m} w_{j}}{\sum_{j=1}^{m} \frac{w_{j}}{f_{j t}}}=\frac{1}{\sum_{j=1}^{m} \frac{w_{j}}{f_{j t}}}
$$

Where, $\hat{y}_{t}$ is the weighted harmonic average comprehensive prediction value at the time $t, \omega_{j}$ is the weighted coefficient of the $j$-th method, and it satisfies the normalized constraint conditions:

$$
\sum_{j=1}^{m} w_{j}=1, w_{j} \geq 0, j=1,2, \cdots m
$$

The formula (5) can be changed to:

$$
\sum_{j=1}^{m} \frac{\omega_{j}}{f_{j t}}=\frac{1}{\hat{y}_{t}}, j=1,2, \cdots, m
$$

Assuming that under ideal conditions, that is $\hat{y}_{t}=y_{t}$, the prediction error does not exist, there are:

$$
\sum_{j=1}^{m} \frac{w_{j}}{f_{j t}}=\frac{1}{y}, t=1,2, \cdots, T
$$

However, in the practice of forecasting, the errors of comprehensive forecasting generally exist. So the prediction error is defined as:

$$
e_{t}=\sum_{j=1}^{G} \frac{w_{j}}{f_{j t}}-\frac{1}{y_{t}}, t=1,2, \cdots, T
$$

$e_{t}$ is called the prediction error of weighted harmonic average comprehensive prediction for time $t$.

Obviously, the smaller the sum of squares of prediction errors is, the better. Therefore, the harmonic average comprehensive prediction model based on the sum of squares of prediction errors as the criterion is the following optimization problems:

$$
\left\{\begin{array}{l}
\min J_{k}=\sum_{t=1}^{n} e_{t}{ }^{2} \\
\text { S.t. } \\
\sum_{j=1}^{m} \frac{w_{j}}{f_{j t}}-\frac{1}{x_{t}}=e_{t}, t=1,2, \cdots, n \\
\sum_{j=1}^{m} w_{j}=1, w_{j} \geq 0, j=1,2, \cdots m
\end{array}\right.
$$

$R_{m}=(1,1, \cdots 1)^{T}$ is called m-dimensional full-1 vector.

$$
\begin{aligned}
e & =\left(e_{1}, e_{2}, \cdots e_{n}\right)^{T} \\
Y & =\left(\frac{1}{y_{1}}, \frac{1}{y_{2}}, \ldots, \frac{1}{y_{n}}\right)^{T} \\
F & =\left[\begin{array}{l}
\frac{1}{f_{11}}, \frac{1}{f_{21}}, \cdots \frac{1}{f_{m 1}} \\
\frac{1}{f_{12}}, \frac{1}{f_{22}}, \cdots \frac{1}{f_{m 2}} \\
\vdots \vdots \\
\vdots \\
\frac{1}{f_{1 n}}, \frac{1}{f_{2 n}}, \cdots \frac{1}{f_{m n}}
\end{array}\right]
\end{aligned}
$$

Then:

$$
\sum_{t=1}^{n} e_{t}^{2}=e^{T} e=(F W-Y)^{T}(F W-Y)
$$

The matrix form is denoted as:

$$
\left\{\begin{array}{l}
\min J=(F W-Y)^{T}(F W-Y) \\
R_{m}{ }^{T} W=1 \\
W \geq 0
\end{array}\right.
$$

\section{Cases and analysis}

The model proposed in this paper uses the forecasting results of a certain area from 2000 to 2009 as historical data. And the model is applied to carry out comprehensive forecasting tests of the regional loads from 2010 to 2013. The model in Table 1 (1) is an 
econometric model, (2) a stepwise regression model, (3) a grey exponential smoothing model, (4) a fuzzy clustering model, (5) a fuzzy linear model. Regression model, (6) is a fuzzy exponential smoothing model, (7) is a grey group model, (8) is a grey moving average model.

The optimal weight coefficients of the models are $0.135808,0.125085,0.06812,0.093,0.276515,0.12$, 0.131827 and 0.04 , respectively.

The forecast for the forecast year is shown in Table 2 .

Table 1. The Real Load and the Prediction Result of Each Model for One Area

(Unit: 104kW)

\begin{tabular}{cccccc}
\hline Year & Real value & 1 & 2 & 3 & 4 \\
\hline 2000 & 1097 & 1089 & 1110 & 1166 & 1149 \\
2001 & 1210 & 1176 & 1182 & 1262 & 1288 \\
2002 & 1356 & 1318 & 1312 & 1366 & 1413 \\
2003 & 1491 & 1478 & 1518 & 1479 & 1527 \\
2004 & 1677 & 1636 & 1650 & 1601 & 1649 \\
2005 & 1838 & 1815 & 1781 & 1733 & 1782 \\
2006 & 1968 & 1979 & 1936 & 1875 & 1925 \\
2007 & 2061 & 2118 & 2095 & 2030 & 2080 \\
2008 & 2130 & 2231 & 2196 & 2197 & 2247 \\
2009 & 2284 & 2330 & 2309 & 2378 & 2427 \\
2010 & 2617 & 2488 & 2598 & 2574 & 2622 \\
2011 & 2773 & 2773 & 2908 & 2786 & 2832 \\
2012 & 3007 & 2946 & 3164 & 3015 & 3060 \\
2013 & 3294 & 3135 & 3379 & 3264 & 3306 \\
\hline Year & Real value & 5 & 6 & 7 & 8 \\
\hline 2000 & 1097 & 1078 & 1049 & 1143 & 1085 \\
2001 & 1210 & 1124 & 1174 & 1246 & 1177 \\
2002 & 1356 & 1275 & 1319 & 1357 & 1277 \\
2003 & 1491 & 1436 & 1499 & 1478 & 1386 \\
2004 & 1677 & 1592 & 1626 & 1608 & 1504 \\
2005 & 1838 & 1760 & 1859 & 1749 & 1631 \\
2006 & 1968 & 1909 & 2002 & 1901 & 1770 \\
2007 & 2061 & 2065 & 2101 & 2065 & 1920 \\
2008 & 2130 & 2206 & 2157 & 2241 & 2084 \\
2009 & 2284 & 2370 & 2202 & 2429 & 2261 \\
2010 & 2617 & 2564 & 2431 & 2631 & 2453 \\
2011 & 2773 & 2762 & 2617 & 2846 & 2661 \\
2012 & 3007 & 2979 & 2931 & 3075 & 2887 \\
2013 & 3294 & 3218 & 3246 & 3317 & 3132 \\
\hline
\end{tabular}

Table 2. Prediction results of harmonic average comprehensive forecasting model

(Unit: 104kW)

\begin{tabular}{ccccc}
\hline Year & Real value & Forecasting & Error & Error (\%) \\
\hline 2010 & 2617 & 2575.43 & -41.57 & -1.59 \\
2011 & 2773 & 2804.27 & 31.27 & 1.13 \\
\hline
\end{tabular}

\begin{tabular}{lllll}
2012 & 3007 & 3038.52 & 31.52 & 1.05 \\
2013 & 3294 & 3281.39 & 31.39 & 0.97 \\
\hline
\end{tabular}

From the forecasting results, the harmonic average comprehensive forecasting model can achieve high fitting accuracy, the maximum forecasting error is about $1.5 \%$, which can better meet the needs of load forecasting.

\section{Conclusion}

Power system load forecasting is the basis of power system planning and operation, and an important part of power market operation. Its accuracy directly affects the overall operation mode of the power market. So far, there have been many achievements in the research of power system load forecasting. The main content of this paper is to use artificial immune algorithm to forecast medium and long-term load of power system.

In this paper, artificial immune algorithm is used to determine the weight coefficients of each scheme. Based on the basic genetic algorithm and combined with the idea of artificial immune algorithm, the weight coefficients are constantly changed by genetic, mutation and selection methods. Finally, the weight coefficients in genes are evaluated by calculating the expected survival rate, and the optimal comprehensive prediction is searched by using the harmonic average comprehensive prediction model. The weight of optimal comprehensive prediction.

Practical examples show that the harmonic average forecasting model for power load comprehensive forecasting based on AIS proposed in this paper can obtain high forecasting accuracy. At the same time, the introduction of population diversity control has greatly improved the efficiency of calculation and search compared with the traditional genetic algorithm, and has the value of further research.

\section{References}

1. Xiao Guoquan, Wang Chun and Zhang Fuwei. Power load forecasting (CEPP, 2001).

2. Liu Meijie, Gu Jie, D\&U, 23(1):19-22(2006).

3. $\mathrm{Yu}$ Jianjun, Zheng Feng, Sun Shudong, CEA, 40 (23): 1-3(2004).

4. Li Chunhua, Zhu Yanfei and Mao Zongyuan. CEA, 40 (22): 84-87 (2004).

5. Jiang Jiping, Ji Fang, CM, 11: 122-126( 2017).

6. An qiuxian, NCEPU, ( 2016)

7. Liu Xiaojuan, Fang Jianan, ECEP, 40(04): 518520(2012). 